\title{
Environmental scan of provincial and territorial planning for COVID-19 vaccination programs in Canada
}

\author{
Shannon MacDonald ${ }^{1 *}$, Hannah Sell ${ }^{1,2}$, Sarah Wilson ${ }^{3}$, Samantha Meyer ${ }^{4}$, Arnaud Gagneur ${ }^{5}$, \\ Ali Assi ${ }^{1}$, Manish Sadarangani ${ }^{6}$, and members of the COVImm Study Team
}

\begin{abstract}
Background: Public health departments in Canada are currently facing the challenging task of planning and implementing coronavirus disease 2019 (COVID-19) vaccination programs.

Objective: To collect and synthesize information regarding COVID-19 vaccination program planning in each province and territory of Canada, including logistic considerations, priority groups, and vaccine safety and effectiveness monitoring.
\end{abstract}

Methods: Provincial/territorial public health leaders were interviewed via teleconference during the early planning stage of COVID-19 vaccination programs (August-October 2020) to collect information on the following topics: unique factors for COVID-19 vaccination, intention to adopt National Advisory Committee on Immunization (NACl) recommendations, priority groups for early vaccination, and vaccine safety and effectiveness monitoring. Data were grouped according to common responses and descriptive analysis was performed.

Results: Eighteen interviews occurred with 25 participants from 11 of 13 provinces/territories $(\mathrm{P} / \mathrm{Ts})$. Factors unique to COVID-19 vaccination included prioritizing groups for early vaccination $(n=7)$, public perception of vaccines $(n=6)$, and differing eligibility criteria $(n=5)$. Almost all $P / T s(n=10)$ reported reliance on $\mathrm{NACl}$ recommendations. Long-term care residents $(n=10)$ and healthcare workers $(n=10)$ were most frequently prioritized for early vaccination, followed by people with chronic medical conditions $(n=9)$ and seniors $(n=8)$. Most $P / T s(n=9)$ are planning routine adverse event monitoring to assess vaccine safety. Evaluation of effectiveness was anticipated to occur within public health departments $(n=3)$, by researchers $(n=3)$, or based on national guidance $(n=4)$.

Conclusion: Plans for COVID-19 vaccination programs in the P/Ts exhibit some similarities and are largely consistent with $\mathrm{NACl}$ guidelines, with some discrepancies. Further research is needed to evaluate COVID-19 vaccination programs once implemented.
This work is licensed under a Creative Commons Attribution 4.0 International License.

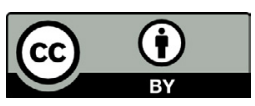

Affiliations

${ }^{1}$ Faculty of Nursing, University of Alberta, Edmonton, $A B$

2 School of Public Health, University of Alberta, Edmonton, $\mathrm{AB}$

${ }^{3}$ Public Health Ontario, Toronto, ON

${ }^{4}$ School of Public Health and Health Systems, University of Waterloo, Waterloo, ON

${ }^{5}$ Département de pédiatrie, Université de Sherbrooke, Centre de Recherche du CHUS, Sherbrooke, QC

${ }^{6}$ University of British Columbia, Vancouver, BC

*Correspondence:

smacdon@ualberta.ca

Suggested citation: MacDonald SE, Sell H, Wilson S, Meyer SB, Gagneur A, Assi A, Sadarangani M and members of the COVImm Study Team. Environmental scan of provincial and territorial planning for COVID-19 vaccination programs in Canada. Can Commun Dis Rep 2021;47(5/6):285-91.

https://doi.org/10.14745/ccdr.v47i56a07

Keywords: COVID-19, vaccination, vaccine, pandemic, vaccination program

\section{Introduction}

The race for the development of coronavirus disease 2019 (COVID-19) vaccines is well underway, with the first vaccines now approved for use in Canada (1). Canadian public health officials are facing the next major challenge of the pandemic: planning and implementing the COVID-19 vaccination programs. Planning has been particularly challenging in comparison with other vaccines given the speed at which vaccine development has occurred, the need to manage multiple new but differing vaccines, and the fact that a large proportion of the population will need to be vaccinated to significantly interrupt the spread of the virus among the general population $(2,3)$. As initial vaccine supply is limited $(3,4)$, one important consideration is 
the prioritization of target groups for COVID-19 vaccination. The National Advisory Committee on Immunization (NACl) has released guidance outlining key populations for receiving initial vaccine supply $(3,5)$. However, it is ultimately up to provincial/territorial governments whether to follow these guidelines, and to determine the logistics of COVID-19 vaccination programs, including vaccine dose allocation, delivery, storage, administration, monitoring and reporting (6). Conversely, the role of the federal government is vaccine approval and procurement, and to provide guidance on vaccine use (6).

It is important to understand and document the processes and strategies that have been employed by each of the provinces/territories (P/Ts) in their COVID-19 vaccination planning. Identifying the range of strategies, highlighting new and innovative approaches, and learning from successful and unsuccessful approaches will enhance our capacity to respond to similar challenges we will undoubtedly face in the future. As such, the objective of this study was to use key informant interviews to collect and synthesize information regarding planned COVID-19 vaccination programs in each of the P/Ts, including logistic considerations, priority groups, and vaccine safety and effectiveness monitoring.

\section{Methods}

This pan-Canadian environmental scan involved structured key informant interviews of public health leaders from $\mathrm{P} / \mathrm{Ts}$ across Canada. The research team included researchers from six $\mathrm{P} / \mathrm{Ts}$, as well as knowledge users from the NACl Secretariat and three P/T health departments. Knowledge users assisted in recruitment, identified topics of value to include in the interview guide, and were provided the study findings for their reference in decisionmaking. The goal of the scan was to capture and synthesize the perspectives of public health leaders actively involved in $\mathrm{P} / \mathrm{T}$ immunization program planning. We recruited $P / T$ members of the Canadian Immunization Committee (CIC), and when they were unavailable to participate, we asked them to designate a replacement. Additional participants were identified through our research team members, $\mathrm{P} / \mathrm{T}$ health departments and the $\mathrm{NACl}$ Secretariat who are knowledgeable of $P / T$ vaccine program leadership. Key informants were contacted via an initial email sent by the NACI Secretariat, inviting them to participate in the study. Interested individuals were emailed an information sheet and consent form. To optimize response rate, up to two email reminders were sent. Some participants were identified through snowball sampling, with study participants suggesting additional key informants. Interviews took place from August to October 2020, prior to release of NACl's preliminary guidance (3) and the approval of any COVID-19 vaccines in Canada. Interviews (35-60 minutes long) were conducted by members of the research team (HS, $A A, M K)$ via teleconference.
Interview questions included key topics related to COVID-19 vaccination, as identified in scientific literature and news articles, and augmented with input from the immunization experts on the research team and knowledge users, including the $\mathrm{NACl}$ Secretariat (see Supplemental material). The structured interview guide consisted of mainly open-ended questions about the following topics: unique factors to be considered in COVID-19 vaccination program planning, the extent of reliance on $\mathrm{NACl}$ recommendations, the use of a geographical prioritization framework for vaccine allocation, target groups for prioritization for early vaccination, and plans for monitoring vaccine safety and effectiveness. The interview guide was reviewed and edited by immunization experts and pilot tested with an individual who worked in provincial immunization program planning, but was not involved in the study, to check face and content validity, flow, and comprehension. The inclusion of multiple perspectives within and between $\mathrm{P} / \mathrm{Ts}$ enhanced the credibility of findings. The guide was shared with participants prior to the interview. Ethical approval for this study was obtained from the Health Research Ethics Board at the University of Alberta.

Interviews were audio-recorded and transcribed verbatim by one member of the research team and any personally identifying information was removed. The same team member then coded and categorized participant responses. Given the very structured nature of the interviews, analysis involved little subjective interpretation. However, to ensure rigor, coding and categorization were validated by three other team members to ensure they accurately reflected and were fully representative of participants' responses. Descriptive analysis of response counts was performed using Microsoft Excel. Participant responses were synthesized and presented by P/T.

\section{Results}

Invitation emails from $\mathrm{NACl}$ were sent to 35 potential participants: 13 agreed to participate; one declined; and 21 did not respond. Twelve participants were recruited from other participants, five through referrals and seven joined the interviews of their colleagues. Therefore, some interviews contained more than one participant. In total, there were 18 interviews with 25 participants from 11 of the 13 P/Ts. Table 1 shows the demographics of the study sample.

\section{Unique factors for COVID-19 vaccination programs}

A wide array of factors that are unique to planning for COVID-19 vaccination programs were identified (see Table 2). Participants from slightly over half of $P / T s(n=7)$ indicated the need to prioritize target groups for early vaccination. Many $P / T s(n=5)$ also highlighted the possibility of having different eligibility 
Table 1: Demographic information of the study sample $(\mathrm{N}=25)^{\mathrm{a}}$

\begin{tabular}{|c|c|}
\hline Characteristic & Number of participants, n \\
\hline \multicolumn{2}{|l|}{ Province/territory } \\
\hline British Columbia & 1 \\
\hline Alberta & 4 \\
\hline Saskatchewan & 3 \\
\hline Manitoba & 4 \\
\hline Ontario & 3 \\
\hline Québec & 3 \\
\hline Newfoundland and Labrador & 1 \\
\hline Nova Scotia & 3 \\
\hline New Brunswick & 0 \\
\hline Prince Edward Island & 1 \\
\hline Nunavut & 1 \\
\hline Northwest Territories & 1 \\
\hline Yukon & 0 \\
\hline \multicolumn{2}{|l|}{ Perspective } \\
\hline Provincial/territorial & 12 \\
\hline Regional/municipal & 9 \\
\hline Both & 4 \\
\hline \multicolumn{2}{|l|}{ Job title } \\
\hline $\begin{array}{l}\text { Director of Immunization or } \\
\text { Communicable Disease Control }\end{array}$ & 2 \\
\hline $\begin{array}{l}\text { Immunization Program or Policy } \\
\text { Manager }\end{array}$ & 7 \\
\hline Medical Officer of Health & 5 \\
\hline Public Health or Medical Consultant & 3 \\
\hline Policy Analyst & 2 \\
\hline $\begin{array}{l}\text { Public Health or Communicable } \\
\text { Disease Specialist }\end{array}$ & 2 \\
\hline Other & 4 \\
\hline
\end{tabular}

criteria for each vaccine (i.e. if one vaccine is more effective in older adults), which may impact the order of priority groups.

Some participants from $\mathrm{P} / \mathrm{Ts}$ also discussed factors related to public engagement, including having clear communication with the public regarding safety implications, eligibility criteria and priority groups ( $n=3 P / T s)$. Likewise, six $P / T s$ highlighted the need to manage public perception of COVID-19 vaccines. Specifically, one $P / T$ felt that vaccine hesitancy for COVID-19 vaccines would be greater than for previous vaccines.

The $\mathrm{P} / \mathrm{Ts}$ also discussed unique factors related to logistics and supply of COVID-19 vaccines. Four P/Ts highlighted the unique storage requirements of some of the vaccines, with some $\mathrm{P} / \mathrm{Ts}$ stating that it was unlikely that all providers currently had the capacity to store vaccines at the appropriate temperature.
Table 2: Provinces/territories' unique factors planned for consideration for COVID-19 vaccination programs $(\mathrm{N}=11)$

\begin{tabular}{c|l}
\hline Unique factor & Number of $\mathrm{P} / \mathrm{Ts}^{\mathrm{a}}$, $\mathrm{n}$
\end{tabular}

Priority groups

\begin{tabular}{|l|l}
\hline Prioritization of target groups & 7
\end{tabular}

Differing eligibility criteria

Equity in delivery

5

Public engagement

Public perception of the vaccine, including vaccine hesitancy

Clear communication with public

Logistics and supply

Logistics, storage, cold-chain management

Limited vaccine supply, availability of vaccine

Availability of PPE and other vaccination supplies (other than the vaccine itself)

Vaccine distribution

Resource issues (in general)

Vaccine procurement

Delivery

COVID-19 related restrictions, public health measures, PPE

Vaccine provider (e.g. physicians,

pharmacists, public health)

Appointment-based delivery versus mass clinics

Need to vaccinate everyone, large volume of people

Less human resources due to COVID-19 redeployment

Training for providers

Uncertainty, not having enough information to plan

Vaccine characteristics

Possibility of needing more than one dose

Vaccine safety

Dealing with a new vaccine

Considerations for the route of administration

Possibility of having more than one vaccine

Speed with which vaccine development is occurring

Abbreviations: COVID-19, coronavirus disease 2019; PPE, personal protective equipment; $\mathrm{P} / \mathrm{T}$, province/territory

a Some $P / T$ responses fell into more than one category

Others noted that supply of the vaccine $(n=3)$ and other vaccination supplies $(n=3)$ will likely be limited.

Planning for the delivery of the COVID-19 vaccines was anticipated to be challenging, with some $P / T s(n=4)$ reporting that they were unsure about which providers would deliver the vaccines (e.g. public health, physicians, pharmacists), or whether 
they would have appointment-based clinics or mass clinics $(n=3)$. Similarly, four $\mathrm{P} / \mathrm{Ts}$ mentioned the need for adapting vaccination clinics to follow COVID-19 recommendations, including physical distancing, personal protective equipment, layout, one-way flow of traffic and ventilation. One $\mathrm{P} / \mathrm{T}$ mentioned including industrial engineers on their planning team to consider these factors. A full list of $\mathrm{P} / \mathrm{T}$ responses is provided in Table 2 .

\section{Reliance on $\mathrm{NACl}$ recommendations}

Almost all $P / T s(n=10)$ indicated that they would likely rely on the $\mathrm{NACl}$ recommendations for target groups in planning their COVID-19 vaccination strategies. One $\mathrm{P} / \mathrm{T}$ indicated that they would more likely rely on their provincial/territorial immunization committee recommendations.

\section{Priority group ranking}

Participants were asked to rank their top five priority groups, with rank 1 representing the group that should receive COVID-19 vaccination first. For reporting purposes, we used the ranking of the respondent from each $\mathrm{P} / \mathrm{T}$ that had the most cross-provincial perspective based on their job position and whether they stated they had a provincial perspective as opposed to regional/municipal. One P/T did not answer, for a total of $10 \mathrm{P} / \mathrm{Ts}$. All of the $\mathrm{P} / \mathrm{Ts}$ ranked long-term care residents $(n=10)$ and healthcare workers $(n=10)$ in the top five priority groups for receiving COVID-19 vaccination. Specifically, six P/Ts indicated long-term care residents as top priority, three indicated healthcare workers and one indicated seniors. Three $\mathrm{P} / \mathrm{Ts}$ ranked healthcare workers second, followed by long-term care residents $(n=2)$, people with chronic medical conditions $(n=2)$, seniors $(n=2)$, and essential workers $(n=1)$. Groups ranked third included seniors $(n=3)$, long-term care residents $(n=2)$, healthcare workers $(n=1)$, people with chronic medical conditions $(n=1)$, people of Indigenous ancestry $(n=1)$, those with socio-economic disadvantage $(n=1)$ and people living in remote communities $(n=1)$. Figure 1 provides a full summary of $P / T$ rankings.

\section{Use of a geographical prioritization framework} None of the $P / T s$ had firm plans for a geographical prioritization framework based on disease incidence (i.e. target groups in high COVID-19 incidence areas are prioritized over target groups in low incidence areas). The majority of $P / T s(n=7)$ were open to this approach if advised by $\mathrm{NACl}(n=1)$, or if the vaccine characteristics $(n=1)$ or number of doses available $(n=3)$ warrants it. Three $\mathrm{P} / \mathrm{Ts}$ were against using a geographical prioritization framework due to concerns with the equity of this approach $(n=1)$ or due to their jurisdiction's small geography or dense population $(n=2)$. One $P / T$ did not know if they were planning on using a geographical prioritization framework.

\section{Monitoring vaccine safety and effectiveness}

With regards to post-market vaccine safety monitoring, most $P / T s(n=9)$ planned to conduct their routine adverse event monitoring, while some $(n=3)$ anticipated enhanced surveillance
Figure 1: Provinces/territories' priority group choices to include in their top five groups to be considered for early vaccination in the presence of limited vaccine supply $(\mathrm{N}=10)^{\mathrm{a}, \mathrm{b}}$

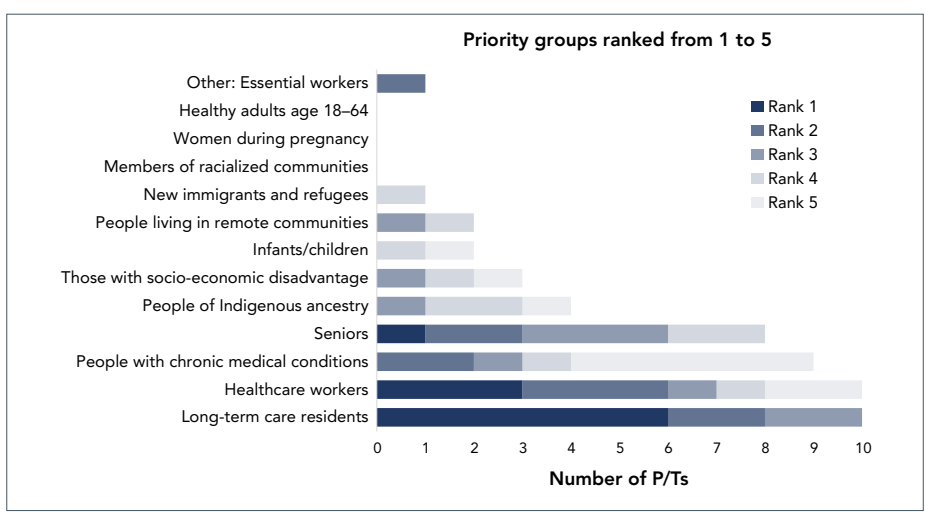

Abbreviation: $\mathrm{P} / \mathrm{Ts}$, provinces/territories

a One province/territory chose not to answer this question

${ }^{b}$ For those who selected seniors $(n=8)$, seven indicated that they would target seniors aged $65+$ years, while one indicated that they would target those $60+$ years

of adverse events (see Table 3$)$. Some $P / T s(n=4)$ anticipated that this will be done by federal/provincial/territorial committees and groups. For post-market vaccine effectiveness monitoring, some $P / T s(n=3)$ anticipated that their $P / T$ public health departments would do this, with a similar number $(n=3)$ stating that this will be routine information collected. Others $(n=3)$ expected that this will be done by researchers or research organizations.

Table 3: Provinces/territories' planned approach to COVID-19 vaccine safety and effectiveness monitoring $(\mathrm{N}=11)$

\begin{tabular}{|l|r|}
\hline \multicolumn{1}{|c|}{ Planned approach } & Number of P/Ts ${ }^{a}, \mathbf{n}$ \\
\hline Safety & 9 \\
\hline Regular adverse event reporting & 3 \\
\hline Enhanced surveillance of adverse events & 4 \\
\hline $\begin{array}{l}\text { Reliance on federal/provincial/territorial } \\
\text { committees and groups (e.g. CIC, CIRC) }\end{array}$ & 1 \\
\hline Reliance on what NACl recommends & 1 \\
\hline Undecided & 2 \\
\hline Do not know & \\
\hline Effectiveness & 3 \\
\hline $\begin{array}{l}\text { Reliance on provincial public health (e.g. } \\
\text { surveillance teams) }\end{array}$ & 3 \\
\hline $\begin{array}{l}\text { Reliance on researchers/research } \\
\text { organizations }\end{array}$ & 4 \\
\hline Reliance on NACl or other national guidance & 3 \\
\hline $\begin{array}{l}\text { Collect routine monitoring information (e.g. } \\
\text { number of clients who tested positive after } \\
\text { vaccination, vaccine coverage) }\end{array}$ & 2 \\
\hline Undecided & 1 \\
\hline Do not know & 2 \\
\hline No answer & $\begin{array}{l}\text { Abbreviations: ClC, Canadian Immunization Committee; CIRC, Canadian Immunization Registry } \\
\text { and Coverage Network; NACl, National Advisory Committee on Immunization; } \\
\text { P/T, province/territory } \\
\text { a Some responses by P/T fall into more than one of the above categories }\end{array}$ \\
\hline
\end{tabular}

CCDR • May/June $2021 \bullet$ Vol. 47 No. 5/6

Page 288 


\section{Discussion}

Although $\mathrm{P} / \mathrm{T}$ rankings of potential priority groups were collected prior to the publication of NACl's guidance documents, the overall $\mathrm{P} / \mathrm{T}$ rankings aligned somewhat with $\mathrm{NACl}$ recommendations. Specifically, the groups ranked highest in this study were healthcare workers and long-term care residents, followed by people with chronic medical conditions and seniors. The most recent $\mathrm{NACl}$ recommendations prioritize healthcare workers, long-term care residents and staff, seniors aged 70 years and older (with those $80+$ years having highest priority) and adults in Indigenous communities (5). The notable difference between the $\mathrm{P} / \mathrm{T}$ rankings in our study and $\mathrm{NACl}$ recommendations is that less than half of $\mathrm{P} / \mathrm{Ts}$ ranked Indigenous communities in the top five prioritized groups, and that people with chronic medical conditions (ranked third by most $\mathrm{P} / \mathrm{Ts}$ ) were not included in NACl's most recent guidance on early vaccination (5).

A common consideration among $\mathrm{P} / \mathrm{Ts}$ was the potential negative public perception of COVID-19 vaccines. Many P/Ts recognized the important role public health will have in the development of communication strategies to counter these concerns. A Statistics Canada survey in June 2020 reported that $76.5 \%$ of Canadians would be very likely or somewhat likely to get a COVID-19 vaccine when available (7), but data from a national Leger survey in November 2020 estimate this number to be $65 \%$ (8).

The $\mathrm{P} / \mathrm{Ts}$ also highlighted the challenging logistics of vaccine delivery and the need to ensure that vaccination clinics follow public health recommendations on distancing, using personal protective equipment, disinfection and ventilation, etc. Multiple $\mathrm{P} / \mathrm{Ts}$ viewed the 2020-2021 seasonal influenza program as a trial for how COVID-19 vaccine delivery may occur. Following the $\mathrm{H} 1 \mathrm{~N} 1$ pandemic, it was noted that well-functioning influenza vaccination programs are essential for ensuring that adequate infrastructure is available for pandemic vaccination response (9). Guidance on strategies for influenza vaccine delivery during the pandemic were provided by $\mathrm{NACl}$ early in the pandemic (10).

Having a unified approach to COVID-19 vaccination in Canada may be beneficial for providing consistent public messaging and clarifying why certain priority groups have been selected for early vaccination. Public communication strategies are important to prevent vaccine hesitancy and mistrust (9). Furthermore, a unified approach to vaccination may improve equity and produce cost-savings (11). Critics of Canada's long-standing provincial and territorial variability in immunization programs and schedules have argued that lack of consistency in eligibility and modes of delivery results in inconsistencies in public messaging which can undermine public confidence when the rationale for differences is unclear $(11,12)$. Conversely, diversity across $\mathrm{P} / \mathrm{Ts}$ enables flexibility to adapt to the unique circumstances of each jurisdiction, given the variation in geography, population, and
COVID-19 cases across P/Ts (13). Although P/Ts will inevitably develop their own plans for COVID-19 vaccination, results from this study suggest that there will likely be many similarities.

\section{Strengths and limitations}

The strength of this study is the wide variety of perspectives that were obtained on COVID-19 vaccination program planning from most $P / T s$. As well, the use of key informant interviews allowed us to gather in-depth perspectives on COVID-19 vaccination program planning in each $\mathrm{P} / \mathrm{T}$. However, as only a few select individuals were interviewed from each $P / T$, the perspectives gathered are not representative of the entire $P / T s$. Furthermore, there may be variation in individual perspectives across a single $P / T$, although the perspectives shared were very consistent within a given P/T. Generalizability may be limited due to the small sample size and non-random sampling. Interviews were conducted during a period when COVID-19 vaccination planning was in its early stages. It will be interesting to follow whether early plans have changed since the release of $\mathrm{NACl}$ guidance documents $(3,5)$.

\section{Implications}

The implementation of COVID-19 vaccination programs in Canada is in the early stages. There is an opportunity to expand on this study's findings through a variety of research avenues, including the assessment of each P/T's finalized COVID-19 vaccination plan, and how variation in vaccination programs ultimately affects vaccine uptake and effectiveness in each P/T.

This study adds to existing literature by synthesizing $\mathrm{P} / \mathrm{T}$ public health perspectives on COVID-19 vaccination programs at a planning stage. Results can inform policymakers and program planners and can assist $\mathrm{NACl}$ in future development of national guidelines. We anticipate that the information in this study will enable $\mathrm{P} / \mathrm{Ts}$ to learn from one another by comparing their approach to COVID-19 vaccination with others across Canada.

\section{Conclusion}

The key informant interview findings show that Canadian $\mathrm{P} / \mathrm{Ts}$ are facing similar challenges in planning for COVID-19 vaccination. The majority will be relying on $\mathrm{NACl}$ recommendations regarding how to allocate limited vaccine supply. Further research is needed to evaluate provincial/territorial COVID-19 vaccination programs once they are implemented.

\section{Authors' statement}

SMacDonald - Conceptualization, methodology, funding acquisition, supervision, formal analysis, writing (original draft, and review and editing) HS - Conceptualization, investigation, data curation, formal analysis, writing (original draft, and review and editing) SW - Conceptualization, methodology, writing (review and editing) 


\section{Authors' statement (continued)}

SMeyer - Conceptualization, methodology, writing (review and editing)

AG - Conceptualization, methodology, funding acquisition, writing (review and editing)

AA - Conceptualization, investigation, data curation, supervision, project administration, formal analysis, writing (review and editing)

MS - Conceptualization, methodology, writing (review and editing)

The content and view expressed in this article are those of the authors and do not necessarily reflect those of the Government of Canada.

\section{Competing interest}

S MacDonald is supported by a salary award from the Canadian Child Health Clinician Scientist Program. MS is supported by salary awards from the BC Children's Hospital Foundation, the Canadian Child Health Clinician Scientist Program and the Michael Smith Foundation for Health Research. MS has been an investigator on projects funded by GlaxoSmithKline, Merck, Pfizer, Sanofi-Pasteur, Seqirus, Symvivo and VBI Vaccines. All funds have been paid to his institute, and he has not received any personal payments.

\section{Acknowledgements}

This was part of a larger project conducted by the COVImm study team, which included the named authors, as well as: $M$ Tunis, K Benzies, J Bettinger, M Driedger, E Dubé, $R$ Humble, M Kiely, N MacDonald, E Rafferty, and J Robinson. We express particular thanks to M Kiely for her assistance with the interviews.

\section{Funding}

This work was funded by the Canadian Institutes of Health Research.

\section{Supplemental material}

Study interview guide

\section{References}

1. Government of Canada. Vaccines for COVID-19: Authorized vaccines. Ottawa (ON): Government of Canada; (updated 2021; accessed 2021-03-05). https://www.canada.ca/en/ health-canada/services/drugs-health-products/covid19industry/drugs-vaccines-treatments/vaccines.html
2. MacDonald NE, Comeau JL, Dubé È, Bucci LM. COVID-19 and missed routine immunizations: designing for effective catch-up in Canada. Can J Public Health 2020;111(4):469-72. DOI PubMed

3. Ismail SJ, Zhao L, Tunis MC, Deeks SL, Quach C; National Advisory Committee on Immunization. Key populations for early COVID-19 immunization: preliminary guidance for policy. CMAJ 2020;192(48):E1620-32. DOl PubMed

4. World Health Organization. WHO SAGE roadmap for prioritizing uses of COVID-19 vaccines in the context of limited supply. Geneva, Switzerland: WHO; 2020 (accessed 2021-03-05). https://www.who.int/publications/m/ item/who-sage-roadmap-for-prioritizing-uses-of-covid-19vaccines-in-the-context-of-limited-supply

5. National Advisory Committee on Immunization. Guidance on the prioritization of initial doses of COVID-19 vaccine(s). Ottawa (ON): Government of Canada; 2020 (accessed 2020-12-14). https://www.canada.ca/en/publichealth/services/immunization/national-advisory-committeeon-immunization-naci/guidance-prioritization-initial-dosescovid-19-vaccines.html

6. Government of Canada. COVID-19 immunization: Federal, provincial and territorial statement of common principles. Ottawa (ON): Government of Canada; 2020 (accessed 2021-03-05). https://www.canada.ca/en/publichealth/services/diseases/2019-novel-coronavirus-infection/ canadas-reponse/covid-19-immunization-federal-provincialterritorial-statement-common-principles.html

7. Frank K, Arim R. Canadians' willingness to get a COVID-19 vaccine: Group differences and reasons for vaccine hesitancy. Ottawa (ON): StatCan; 2020 (accessed 2020-12-11). https://www150.statcan.gc.ca/n1/pub/45-28-0001/2020001/ article/00073-eng.htm

8. Leger. North American tracker. Montreal ( $\mathrm{QC}$ ): Leger; 2020 (accessed 2020-12-11). https://leger360.com/wpcontent/uploads/2020/11/Legers-North-American-TrackerNovember-30th-2020-min.pdf?x43558

9. Sultana J, Mazzaglia G, Luxi N, Cancellieri A, Capuano A, Ferrajolo C, de Waure C, Ferlazzo G, Trifirò G. Potential effects of vaccinations on the prevention of COVID-19: rationale, clinical evidence, risks, and public health considerations. Expert Rev Vaccines 2020;19(10):919-36. DOI PubMed

10. National Advisory Committee on Immunization (Advisory Committee Statement). Canadian Immunization Guide Chapter on Influenza and Statement on Seasonal Influenza Vaccine for 2020-2021. Ottawa (ON): PHAC; 2020 (accessed 2020-12-15). https://www.canada.ca/content/dam/ phac-aspc/documents/services/publications/healthy-living/ canadian-immunization-guide-statement-seasonal-influenzavaccine-2020-2021/naci-2020-2021-seasonal-influenza-stmteng.pdf

11. Macdonald N, Bortolussi R. A harmonized immunization schedule for Canada: A call to action. Paediatr Child Health 2011;16(1):29-31. DOI PubMed 
12. A patchwork policy: vaccination in Canada. CMAJ 2003;168(5):533. PubMed
13. Government of Canada. COVID-19 daily epidemiology update. Ottawa (ON): Government of Canada; 2021 (accessed 2021-03-09). https://health-infobase.canada.ca/ covid-19/epidemiological-summary-covid-19-cases.html

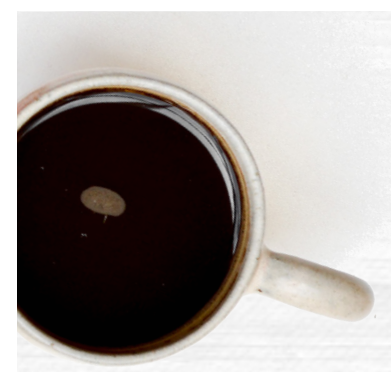

\section{Get CCDR delivered to your inbox}

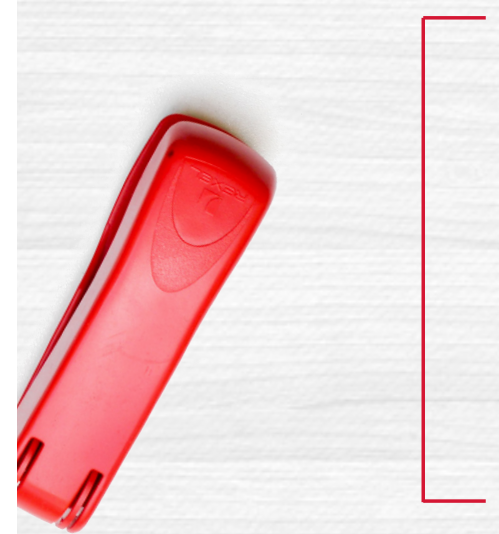

- Know the trends

- Get the testing guidelines

- Stay current on new vaccines

- Learn about emerging infections

- Get the table of contents straight to your inbox

\section{SUBSCRIBE TODAY}

Web search: CCDR+Subscribe Q 